\title{
Transcription Factor Affects Patterning of Axon migration in Caenorhabditis elegans
}

\author{
Received :21/2/2018
}

Accepted : 26/6/2018

\author{
Aseel Raheem Mardan \\ Department of Biology, College of Education; Al-Qadisiya University \\ asrama78@yahoo.com
}

\begin{abstract}
Axon navigation through nervous system development is a chalinging process. How do axons navigate to their final targets still unclear. Several axon guidance cuesresponspel for regulating axon guidancehave been identified in the past decade. Resent studies indicate that transcription factors have a crusial role in the nervous development by coordinatiing neuron response to guidance cues. The nervous system is one of the most complex system that controls the behavior and physiology of body. Caenorhabditis elegans nervous system development involves cell migration and axon guidance. Neuronal cell body and its axon migrate along the dorsal ventral and anterior posterior axis to reach their final position to generate the final nervous system structure. UNC-53 is a cytoplasmic protein that regulates growth cone migration. The C. elegans homolog, NAV 2, a member of the Neuron Navigator protein family, also plays roles in axon guidance and outgrowth.

In this study, it was used the nematode C.elegans to study the transcriptional regulation of axon guidance, axon branching and cell position. It was found unc5(e53) and unc-53(n152) single mutants had less axon outgrowth defects, compared with zag-1(zd86);unc-5(e53) and zag-1(zd86);unc-53(n152) double mutants that displayed a much higher frequency of HSN axon outgrowth defects relative to wild type.
\end{abstract}

Keywords: Axon outgrowth, zag-1 transcription factor, cell migration, Caenorhabditis elegans. 


\section{Introuduction}

Understanding of transcription factors control axon guidance may led to a new approaches to understand neuronal development in vertebrates and invertebrates. Recently, transcription factors can regulate axon guidance process by regulating the genes and function which directly determine the responses of neurons to axon guidance cues and neurotransmitters in the vertebrate and invertebrates nervous system [1], indicating that the transcription factor might influence axon guidance decisions. Nevertheless, how transcription factors control guidance cues is still unknown. Multiple nervous system human disorders and mental retardation diseases are related to abnormal proliferation, migration and axon outgrowth such as autism [2,3], and Alzheimer's disease[4].

The axon guidance is highly regulated process that guides the axons from their origin to their targets area. The neurons extend an axonal processes into specific directions and direct the outgrowth activity into their final positionduring neuronal development [5]. In this study it was consedered that the transcription factor acts as master regulator of this process. The detection of guidance cues occurs at the highly sensitive structure located at the leading edge of the growing axons called the growth cone, which is visualized by Raymon Y. Cajalat the first time[6]. The structur of growth cone became a major interests in neurosciencedevelopment field. Structurally, the growth cones composed of different cytoplasmic structures, including lamellipodia and filopodia. Together, Lamellipodia and filopodia control growth cone movement[7]. Axon branching as axon guidance is important for neuronal structure and function. They are share molecular mechanism[8]. For instance, the guidance molecules that guide axons towards or away their targets have been implicated in enhancing or inhibiting branching of axons[9].

In the past few years several molecular and genetic researches have led to discover four families of canonical guidance molecules that are involved in axon guidance process, including UNC-6/ Netrin, and its receptors, UNC-5/UNC-5 and UNC40/DCC, Slit and its receptor Robo, 
semaphorins/Plexin and Ephrins.

These axon guidance cues are highly conserved and they have been studied in invertebrate and vertebrate systems[3,5, 10]. Netrin issmall family of conserved axon guidance cues. They play an important role in regulating axon guidance toward the ventral midline[7]. The deleted colorectal cancer (DCC/UNC-40) receptor mediates Netrin attraction, whereaes UNC-5/UNC-5 receptor mediates Netrin repulsive[11]. Aditionally, adaptor protein,UNC-53(NAV-2), is a cytoskeletal binding protein uncoordinated phenotype that controls growth cone extension and axon migration during $C$. elegance development. It has a role in remodeling actin cytoskeleton at the leading edge of cell[12].

\section{ZAG-1 Homeodomain Transcription}

\section{Factor}

zag-1 is highly conserved homeodomain transcription factor (Clark and Chiu, 2003; Wacker et al., 2003). Structurally, zag- 1 has five C2H2-type zing finger domains: two zing finger domains at the $\mathrm{N}$-terminal domain and three zing finger domains at the C-terminal domain as well as a single homeodomain in the middle followed by $\mathrm{CtBP}[13]$. The N-terminal and C-terminal zinc finger domains are same. zag-1 gene has roles in regulating multiple aspects of neurons differentiation and nervous system development such as cell position, gene expression, axon guidance and axon branching defects of many interneuron and motor neuron. ZAG-1 is required for HSN differentiation and it acts as first transcription factor that regulates neuron development by enhancing the formation of axon branches[13]. In contrast, zag-1 mutants cause axon pathfinding defects in HSN and AVM morphology and cause severe defects[13].

\section{The C. elegans HSNs and AVM in} Studying Axon Guidance and Branching

To understand axon outgrowth and axon guidance process, it was focused in this study on HSN (hermaphroditespecific motorneuron), AVM (Anterior ventral microtubule), and ALM (Anterior lateral microtubule) $C$. elegans neurons. The HSN neuron is drived egg laying. It is a good for our studies to study the role of molecules in axon guidance pathway because the HSN cell body, which located in midbody, is large to visualize with 
transgenic marker with florescence microscope[14].

The AVM neuron located at the mid ventrally to reach ventral nerve cord and then migrate in an anterior direction toward the nerve ring [15]. anterior. It sends axon to migrate

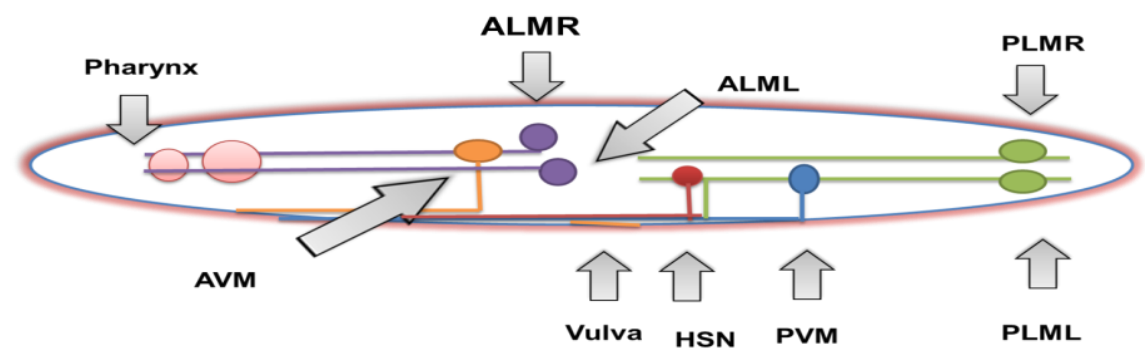

Figure 1. Neurons have Different Patterns of Outgrowth in C. elegans. Adapted from [19].

\section{Materials and Methods}

\section{Cultur of C.elegans}

Bristol strain N2 was used as a wild type. Worms were cultured agar plates seeded with special strain of Eschericiacoli(OP50) as food. Each liter of neurites agar was composed of $3 \mathrm{~g}$ of $\mathrm{NaCl}, 17 \mathrm{~g}$ agar, $2.5 \mathrm{~g}$ Pepton. The nutrint agar was adjusted to $\mathrm{PH}$ 7.0 and autoclave before being poured into the $60 \mathrm{~mm}$ plates. Agar plates stored in a dray and a cold plase after solidifying. After that, one drop of OP50 bacteria was seeded, spread onto the plate and allowed to grow at the room temprature overnight.
All C. elegans strains were incubated and handled at $20^{\circ} \mathrm{C}$ by general techniques as described by Brenner, 1974 [18]. Transfer of worms could be performed by transferring a single worm using a worm picker (a 10 $\mathrm{mm}$ platinum wire mounted on a Pasterior Pipette) or by cutting a small block of agar and transferring the agar block to the new plate.

To examine genetic interactions between zag-1 and other genes, It was looked at animal phenotype of the single, double and triple mutants. If the double and triple mutants phenotypes show the same single mutant phenotype, these two or three genes work in the same pathway. In contrast, 
if the double and triple mutants phenotypes show the different single mutant phenotype, these two or three genes work in different pathways.

The following mutations and transgenes were used in this study:

LGI,unc-40 (e1430), zdIs5 [mec$4: \because G F P]$, LGII, unc-53(n152), LGIV,unc-5(e53), zag-1(zd85), zag1(zd86), kyIs262 [unc-86::myr GFP;odr-1::dsRed], zdIs13 [Ptph$1: \because g f p]$, LGX, unc-6(ev400).

\section{Genetic Crosses of C. elegans}

All crosses are carried out by putting young adult hermaphrodites together with a young adult N2 males from parental strains on a mating plates to increase the mating chance. Usually 10 young adult males and 5 adult hermaphrodites were transformed onto a mating plate. All crosses were performed at $20^{\circ} \mathrm{C}$ and 10 of the resulting F1 heterozygous hermaphrodite were transferred into individual plates. These hermaphrodites were allowed to selffertilize. All genetic crosses were performed under dissecting microscope at $40 \mathrm{X}$ magnification.

\section{Analysis of the HSN Phenotypes}

HSN neurons morphology was visualized by using expression of kyIs262 [unc-86::myr-GFP] and zdIs13 [Ptph-1::gfp] transgenic strains. After eggs hatching, the L1 larvae fed and grew to specific larvae stage. The larvae stage was determined by examining the gonad cell number and gonad size by using differential interference contrast (DIC) microscopy.

Axon migration defects were scored in L4 larval stage. Larval staging determined by the gonad cell number. The L4 larvae mounted in M9 buffer with $10 \mathrm{mM}$ levamisole on a $5 \%$ agarose pad. The worm numbers with ventral phenotypes was counted and calculated the percentage of them from the total measured sample population. Standard error of the mean was used. By using epifluorescent microscopy with a Zeiss $63 \mathrm{X}$ water immersion objective, the images were taken.

\section{Analysis of the AVM Extension Phenotypes}

To analyze AVM axons branching phenotype, the adult worms were mounted on a $5 \%$ agarose pad with 10 mM levamisole buffer. The AVM was visualized by using the expression of 
the zdIs5 [Pmec-4::GFP] transgenic strain under epifluorescent microscopy. The anterior extension defects was scored if the AVM axons did not extend anteriorly, past the nerve ring. The images were taken by using epifluorescent microscopy with a Zeiss $63 \mathrm{X}$ water immersion objective.

\section{Analysis of HSN Cell Body Position}

HSN neurons were visualized using expression of the transgene kyIs262[unc-86::myr-GFP] and zdIs 13 [Ptph-1::gfp] transgenic strains. The larval stage was determined by using differential interference contrast (DIC) microscopy to examine the gonad cell number and gonad size. Staged nematodes larvae were mounted on a $5 \%$ agarose pad with $10 \mathrm{mM}$ levamisole buffer. Images were taken using epifluorescent microscopy with a Zeiss $63 \mathrm{X}$ water immersion objective.

\section{Results}

\section{ZAG-1 Controls the Pattering of HSN Axon Outgrowth Extension}

In this study, an attempt to study the role of zag-1 mutant in axon extension from HSN neuron cell body. In wild type, the HSN axon extends ventrally from the cell body toward the ventral nerve cord and then turns anteriorly towards the nerve ring and grows around the vulva [16].

In L4 larvae, it was found a different patterns of HSN axon extension from the cell body in mutations. Double mutants were made with $u n c-5$ and with $u n c-53$. It was found $u n c-5(e 53)$ and $u n c-53(n 152)$ single mutants had less axon outgrowth defects, while in zag-1(zd86); unc5(e53) and zag-1(zd86);unc-53(n152) double mutants, the HSN axons displayed a much higher frequency of axon outgrowth defects relative to wild type. However, the percentage of the axon outgrowth defects in unc-5(e53) and unc-53(n152) single mutants is low, a zag-1 mutant was shown to enhance the axon outgrowth defects of unc-5(e53) and unc-53(n152). These double mutants had approximately double the percentage of overall axon outgrowth defects compared to the unc-5(e53) and unc-53(n152) single mutants (Figure 2). Since zag-1(zd86) mutants enhanced the outgrowth defects of unc-5(e53) and unc53(n152) mutants, it supports the idea that these genes function in the different pathway to regulate axon outgrowth patterning. 


\section{AL-Qadisiyah Journal of pure Science Vol.23 No.3 Year 2018}

Thus, the effect of UNC-5 and UNC-53 on axon outgrowth requires the presence of ZAG-1, but ZAG-1 also has UNC-5 and UN-53 A

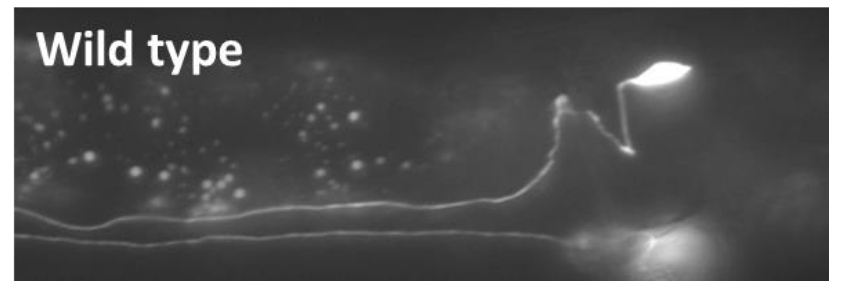

B
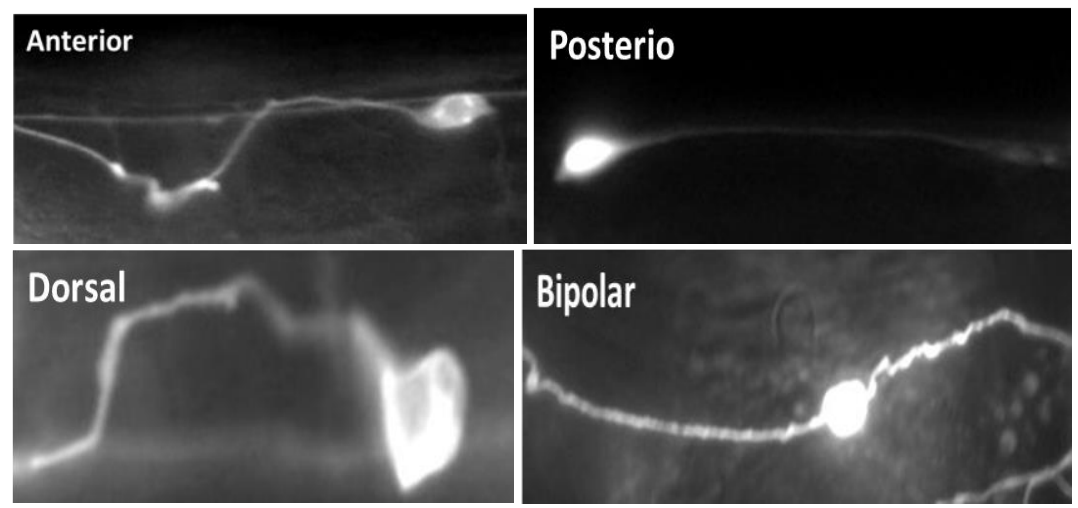

Figure 2. Ventral Axon Extension from the HSN Cell Body

(A) In wild type, the HSN neurons migrates ventrally. (B) In zag-1 mutants, the defects include axons that migrate anteriorly, posteriorly, dorsally or have extra extension. In zag-1 mutants, the ventral axon outgrowth direction decreases compared with ZAG-1 Controls the Pattering of AVM Axon Anterior Extension

Patterning AVM axon anterior extension was checked. In wild type, independent axon outgrowth defects. These data taken together indicate that ZAG-1 is a novel effector of UNC-5 and UNC-53. 


\section{AL-Qadisiyah Journal of pure Science Vol.23 No.3 Year 2018}

interactions involving unc-5, unc-40 and zag-1 do not affect outgrowth patterning at this position (Figures $3 \mathrm{C}$ ) compared to wild type.

A

B
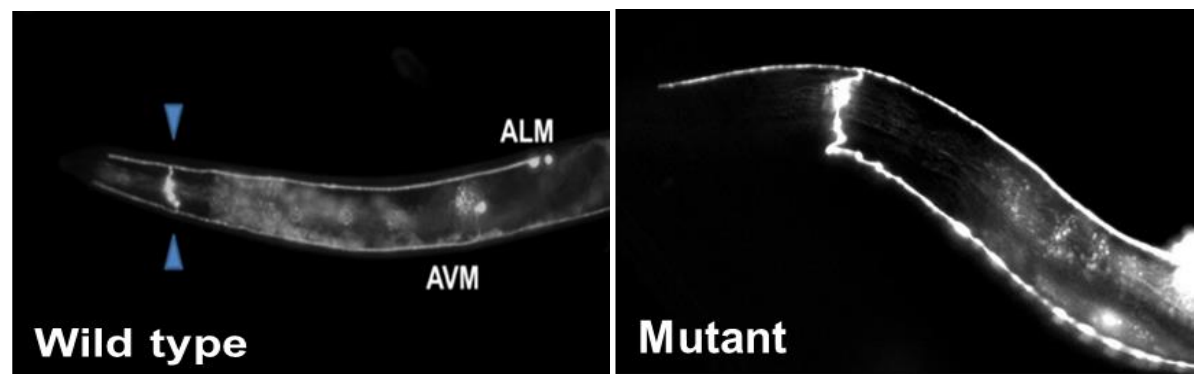

C

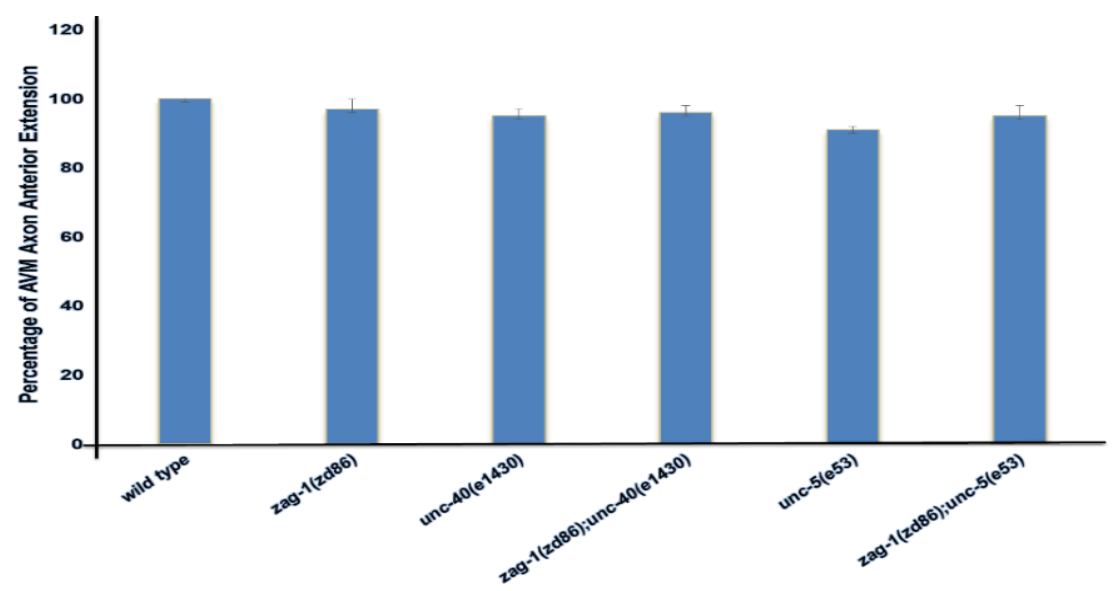

Figure 3. Role of ZAG-1inthe AVM Anterior Extensions Patterning

(A) Photomicrographs of the AVM neuron at the L4 stage in wild-type animals and mutants showing different patterns of axon outgrowth extension. In wild type a single axon travels anteriorly to the nerve ring (arrowheads). At the nerve ring the axon branches; one branch extends further anteriorly and the other extends into the nerve ring. (B) In mutants, axons will only extend into the nerve ring and will not extend anteriorly. (C) The percentage of AVM neurons extend anteriorly, past the nerve ring. 


\section{AL-Qadisiyah Journal of pure Science Vol.23 No.3 Year 2018}

The neuron often extend anteriorly in the $z a g-1(z d 86)$ single mutants, also it does extend anteriorly in the unc5(e53), unc-40(e1430), zag-

A
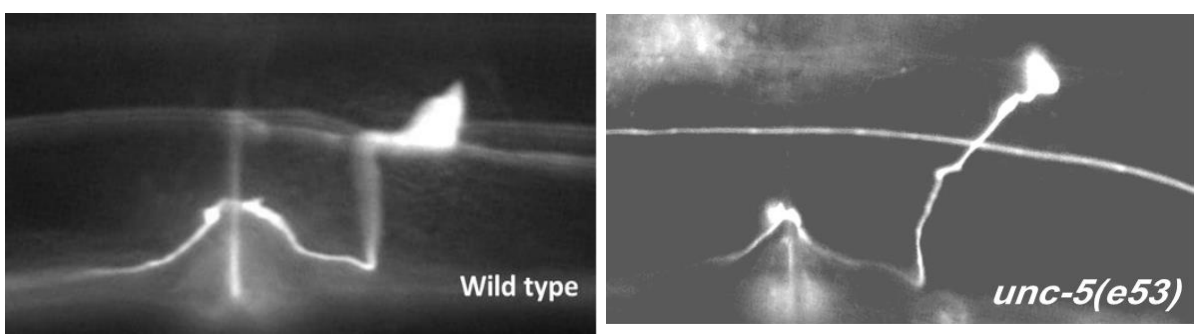

C

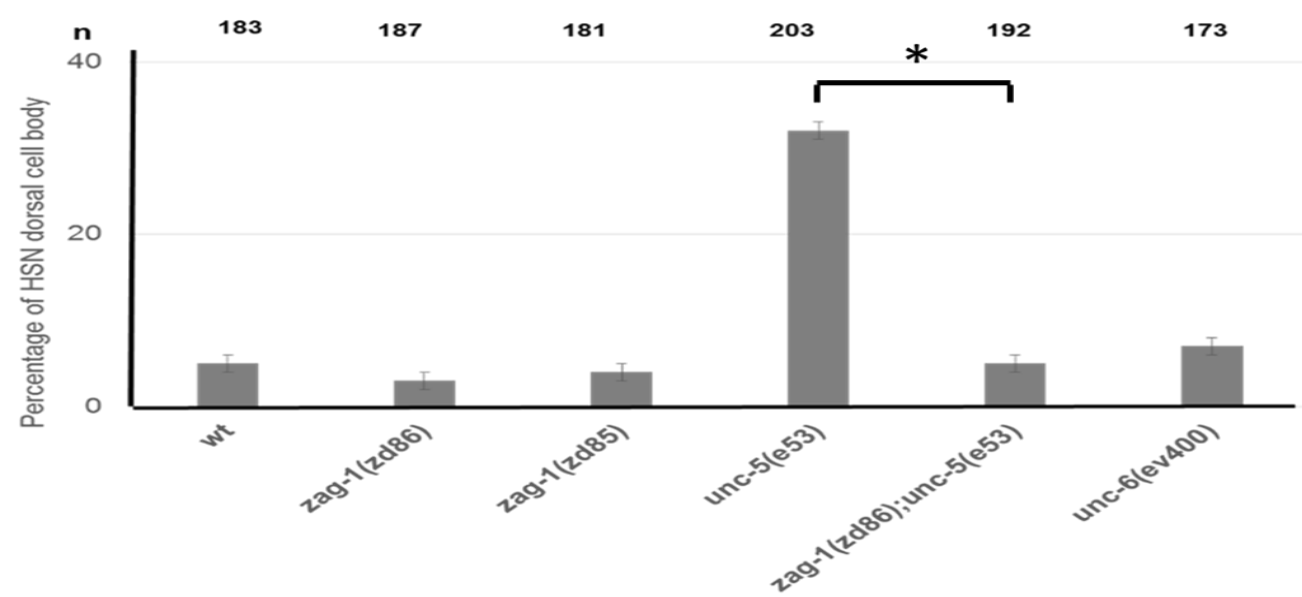

1(zd86);unc-40(e1430) and zag$1(z d 86)$;unc-5(e53)mutants. There is no a significant difference between these mutants and wild type.

B

Figure 4. Photomicrographs of HSN adult stages in wild-type and unc-5(e53) mutants.

(A) In wild-type animal, there is short ventral migration of the HSN cell body. zag-1(zd86) single mutant has wildtype phenotype in HSN cell body position. (B) In unc-5(e53) single mutants, the position of the cell body remains dorsal with a single longer ventral extension, whereas in zag1(zd86);unc-5(e53) double mutants, the position of the HSN cell body migrate ventrally. (C)The percentage of HSN neurons with a cell body mispositioned dorsally. In mutants the HSN cell body often remains in dorsal position. Error bars indicate the standard error mean; $\mathrm{n}$ values are indicated above each column. The student t-test was used to determine if 
there are significant differences

\section{Discussion}

The nervous system function requires that axons grow into the correct target areas. Studies in motor and sensory neurons in vertebrates have been demonstrated the functional relationship between transcription factor and axon guidance. It will be of great interest to identify the targets that explain how transcription factor regulates neuronal morphology in $C$. elegans. It was found that the unc-3 transcription factor can regulate axon guidance [17]. Although this study has focused on the role of transcription factor in axon guidance and branching, it is important to note that the transcription regulation is essential to complete this process.

In this study results showed that ZAG-1 regulates axon outgrowth extension. zag-1 gene acts as a transcriptional repressor to regulate several neuron characteristics like cell position, axonal development and biosynthetic enzyme [20].

zag-1 mutants cause severe axon outgrowth defects in HSNs [13]. It was showed that zag-lmutations alter patterns of HSN outgrowth extension. between strains, $\quad * \mathrm{p}<0.05$. Furthermore, zag-1 mutants cause several defects in sensory neurons [13]. Whereas, it was showed that zag-1 mutants does not have effects on the pattern of anterior outgrowth extension of the AVM neuron.

Studying cell migration and axon guidance in vivo in vertebrates is challenging. C. elegans is a model organism that has used to identify of multiple guidance cues and their receptors. The signaling pathways that are directed cell migration and axon guidance in $C$. elegans are highly conserved in vertebrates.

During the L2 larvae stage in wild type animals there is a short ventral migration of HSN cell body. In this study it was showed that ZAG-1 regulates the HSN cell body position. In zag-1(zd86), the cell body migrate ventrally. It was further observed that in unc-5(e53) mutant, the cell body fail to migrate ventrally. Whereas in zag-1(zd86); unc-5(e53) double mutant, there is ventral migration of HSN cell body. The observations indicate that unc-5 loss of function affect the axon outgrowth pattering and can be enhanced by zag- 1 . 


\section{Acknowledgments}

I would like to thank the Caenorhabditis Genetics Center for strains. I thank Dr. William Wadsworht and Dr. Martha Soto, Department of Pathology and Laboratory Medicine, Rutgers Robert Wood Johnson Medical School, Piscataway, USA for their helpful discussions.

\section{References}

1-Polleux, F., Ince-Dunn,G., Ghosh, A. (2007). Transcriptional regulation of vertebrate axon guidance and synapse formation. Nature Reviews Neuroscience, 8, 331-340.

2- Quinn, C. C., and Wadsworth, W.G. (2008). Axon guidance: asymmetric signaling orients polarized outgrowth. Trends Cell Biol, 18, 597603.

3-Yaron, A., \&Zheng, B. (2007). Navigating their way to the clinic: emerging roles for axon guidance molecules in neurological disorders and injury. Developmental Neurobiology, 67, 1216-1231.

4-Zhang, J. and Cai, H. (2010). Netrin-1 prevents ischemia/reperfusion-induced myocardial infarction via a DCC/ERK1/2/eNOSs1177/NO/DCC feed-forward mechanism. J Mol Cell Cardiol, 48, 1060-1070.

5- Dickson, B. J. (2002). Molecular mechanisms of axon guidance. Science, 298, 1959-1964.

6-Cajal S. R. Y. (1890). A quelle epoqueapparaissent les expansions des cellule nerveuses de la moellee pinere du poulet. Anat. Anz, 5: 609-613.

7-Huber, A.B., Kolodkin, A.L., Ginty, D.D. and Cloutier, J.F. (2003). Signaling at the growth cone: ligand-receptor complexes and the control of axon growth and guidance. Annu Rev Neurosci, 26, 509-563.

8-Dent, E. W., \&Gertler, F. B. (2003). Cytoskeletal dynamics and transport in growth cone motility and axon guidance. Neuron, 40, 209-227.

9-Dent E. W., Barnes A. M., Tang F., Kalil K. (2004). Netrin-1 and semaphorin 3A promote or inhibit cortical axon branching, respectively, by reorganization of the cytoskeleton. J. Neurosci, 24, 3002-12.

10-Tessier-Lavigne, M. and Goodman, C.S. (1996). The molecular 
biology of axon guidance. Science, 274, 1123-1133.

11-Norris AD, Lundquist EA. (2011).UNC-6/netrin and its receptors UNC-5 and UNC-40/DCC modulate growth cone protrusion in vivo in $\mathrm{C}$. elegans. Development, 138, 4433-42.

\section{2-Stringham, E. G. and Schmidt, K.}

L. (2009). Navigating the cell: UNC53 and the navigators, a family of cytoskeletal regulators with multiple roles in cell migration, outgrowth and trafficking. Cell AdhMigr, 3, 342-346.

13- Clark, G. and Chiu, C. (2003). C. elegans ZAG-1, a Zn-fingerhomeodomain protein, regulates axonal development and neuronal differentiation. Development, 130, 3781-3794.

14-Desai, C., Garriga, G., McIntire, S.L., and Horvitz, H.R. (1988). A genetic pathway for the development of the Caenorhabditis elegans HSN motor neurons. Nature, 336, 638-646.

15-Chalfie, M., and Sulston, J. (1981). Developmental genetics of the mechanosensory neurons of Caenorhabditis elegans. DevBiol, 82, 358-370.
16- Adler, C. E., Fetter, R. D. and Bargmann, C. I. (2006). UNC6/Netrin induces neuronal asymmetry and defines the site of axon formation. Nat Neurosci, 9, 511-518.

17-Prasad B.C., et al. (1998). unc-3, a gene required for axonal guidance in Caenorhabditis elegans, encodes a member of the $\mathrm{O} / \mathrm{E}$ family of transcription factors. Development, 125, 1561-1568.

18- Brenner, S. (1974). The genetics of Caenorhabditis elegans. Genetics, 77(1), 71-94.

19- Kulkarni, G., Zhennan $X$., Ahmed M. M., Haichang, L., Xia T., Gerard, L. and William, W. G. (2013). Experimental evidence for UNC-6 (netrin) axon guidance by stochastic fluctuations of intracellular UNC-40 (DCC) outgrowth activity. Biology Open, 2(12), 1300-1312.

20- Wacker, I., Schwarz, V., Hedgecock, E.M. and Hutter, H. (2003). zag-1, a Zn-finger homeodomain transcription factor controlling neuronal differentiation and axon outgrowth in C. elegans. Development, 130(16), 3795-3805. 
AL-Qadisiyah Journal of pure Science Vol.23 No.3 Year 2018 